INNOVACIÓN

\title{
El curso basal de competencias comunicativas en Derecho: Enseñanza de la escritura académica desde un enfoque mixto e interdisciplinar
}

\author{
O curso basal de competências comunicativas em Dereito: \\ Ensino da escrita acadêmica desde um enfoque misto e interdisciplinar \\ The core course of communication skills in Law: \\ Academic writing teaching from a mixed and interdisciplinary approach
}

\author{
Enrique Sologuren Insúa
}

Universidad de Valparaíso y Universidad de los Andes, Chile

Cristina Bonifaz Reyes iD

Universidad de Valparaíso, Chile

\section{Carmen Gloria Núñez Castillo}

Universidad de Chile y Universidad de Playa Ancha, Chile

\begin{abstract}
RESUMEN Este artículo da cuenta de una propuesta intensiva de formación en competencias comunicativas destinada a estudiantes de primer año de la carrera de Derecho de una universidad chilena en el marco de un programa de alfabetización académica y profesional. Presenta la plataforma teórica, metodológica y didáctica de la propuesta centrada en la relevancia de la escritura como herramienta epistémica y la integración de dos dispositivos institucionales de carácter concomitante. Asimismo, analiza el diseño del curso basal al interior de la comunidad de práctica concernida. El curso se proyecta como un espacio formativo donde los estudiantes puedan optimizar sus procesos de producción escrita, transformándose en comunicadores más eficientes. Con esto se concluye que, en el contexto de la educación superior chilena, el curso basal se instala como un dispositivo clave para integrar formación general y orientación disciplinar al promover en su quehacer pedagógico la lectura y escritura de textos disciplinares y el aprendizaje colaborativo a través de la incorporación de mentorías de estudiantes avanzados de las mismas comunidades. También el curso sitúa al estudiante en los textos que
\end{abstract}


efectivamente lee y escribe en esta facultad, propiciando una inserción a los tipos de interacciones y los desafíos del discurso académico y profesional.

PALABRAS CLAVE Curso basal, escritura académica, aprendizaje colaborativo, trabajo por proyectos, géneros discursivos de la comunidad del Derecho.

RESUMO Este artigo analisa uma proposta intensiva de formação em competências comunicativas destinada a estudantes do primeiro ano do curso de Direito no marco de um programa de alfabetização acadêmica e profissional. Para cumprir com este propósito, se revisam os úteis conceituais que sustentam a relevância da escrita como ferramenta epistêmica, se propõe a integração de dois dispositivos institucionais de caráter concomitante, se constrói una plataforma teórica, metodológica e didática; e se analisa o desenho e instalação do curso basal ao interior da comunidade de prática concernida. $\mathrm{O}$ curso é projetado como um espaço de treinamento onde os alunos podem otimizar seus processos de produção escritos, tornando-se comunicadores mais eficientes. Isso conclui que, no contexto do ensino superior chileno, o curso básico é instalado como um dispositivo fundamental para integrar a educação geral e orientação disciplinar, promovendo a leitura e a escrita de textos disciplinares e a aprendizagem colaborativa através de seu trabalho pedagógico e a incorporação de mentorias de estudantes avançados das mesmas comunidades. O curso também coloca o aluno nos textos que realmente leem e escrevem nessa faculdade, promovendo uma inserção aos tipos de interações e aos desafios do discurso acadêmico e profissional.

PALAVRAS-CHAVE Curso basal, escrita acadêmica, aprendizagem colaborativa, trabalho por projetos, gêneros discursivos da comunidade de Dereito.

ABSTRACT This article sets out a thorough proposal based on communication skills instruction aimed at freshmen students who belong to the law degree course in which a program of academic and professional literacy is inserted. To fulfill this purpose, the conceptual framework that supports the importance of writing as an epistemic tool is reviewed. Furthermore, the integration of two concomitant institutional procedures is suggested, as well as the construction of a theoretical, methodological and didactic platform. Finally, the design and installation of a core course within the community of practice concerned is analyzed. The course is projected as a training space where students can optimize their written production processes, becoming more efficient communicators. This concludes that in the context of Chilean higher education, the basal course is installed as a key device to integrate general education and disciplinary guidance by promoting the reading and writing of disciplinary texts and collaborative learning through their pedagogical work. The incorporation of mentorships of advanced students from the same communities. The course also places the student to the texts that read and write in this faculty, promoting an insertion to the types of interactions and the challenges of academic and professional discourse.

KEYWORDS Core course, academic writing, collaborative learning, discourse genres of the Law Community, project work. 


\section{Introducción}

En general, en nuestro contexto universitario nacional, las competencias de lectura, escritura y oralidad no se encuentran insertas de manera curricular, pese a ser habilidades esenciales para la vida académica y profesional (Harvey, 2008; Marinkovich, Sologuren y Shawky, 2018). Tal como plantea González-Álvarez (2016), tradicionalmente han sido dos las formas de abordar el problema de la enseñanza de la escritura en la universidad: desde la formación general, que conceptualiza la escritura como habilidad única transferible a diferentes contextos o desde la orientación disciplinar que asume que la producción escrita es altamente específica, progresiva y dependiente de las comunidades de práctica. El curso basal (Ávila Reyes, González-Álvarez y Peñaloza Castillo, 2013) se trata de una propuesta de formación intensiva en competencias comunicativas con foco en escritura que posee un carácter mixto, a la vez general y disciplinar, lo que posibilita el desarrollo de un trabajo colaborativo e interdisciplinario dentro de un espacio curricular especializado en la comunicación en lengua materna. De esta manera, los estudiantes reciben entrenamiento informado en nociones generales de escritura académica e inician su incorporación paulatina a las prácticas situadas y a las convenciones retóricas propias de cada comunidad, en este caso del derecho (Condon y Rutz, 2013).

En efecto, autores como Shwom y Hirsch (1999) evidencian que la inclusión de acciones curriculares que aborden explícitamente la escritura en términos disciplinares (por ejemplo, a través de una enseñanza interdisciplinar que aúne contenidos lingǘsticos con proyectos de la carrera) pueden promover en los alumnos una conciencia de la importancia y del rol de la comunicación en la formación académica y profesional, así como incentivar el desarrollo de las habilidades de lectura, escritura y oralidad de forma integrada y concomitante (Sologuren, 2015).

La propuesta formativa que aquí se analiza se diseñó durante los años 2013 y 2014 y entra en vigor en el programa de estudio de la carrera desde el año 2015; luego de las fases de prepilotaje y pilotaje en el año 2016 se incorporan al curso las mentorías. El objetivo de este artículo es dar cuenta del diseño y la fundamentación del dispositivo pedagógico curso basal de comunicación académica en la Escuela de Derecho de la Universidad de Valparaíso en el marco de la renovación curricular del pregrado y la instalación del nuevo proyecto educativo institucional, así como la vinculación de este espacio curricular con el Programa de Aprendizaje Colaborativo UV (PAC), que contempla la acción tutorial a través del apoyo integral de pares disciplinares. Asimismo, la metodología de enseñanza-aprendizaje predominante es el aprendizaje basado en proyectos (Stoller, 2002; Tejeda y Sánchez, 2013; Lima y Noya, 2018), lo que permite desarrollar el ciclo completo de proposición de una iniciativa de ley, afianzando el lenguaje académico y profesional a partir de las exigencias de la tarea investigativa-legislativa. Esta plataforma entronca así con un conjunto de instrumen- 
tos didácticos y evaluativos que posibilitan una evaluación orgánica de la escritura académica y una comprensión más profunda del discurso académico en formación dentro de la Facultad de Derecho y Ciencias Sociales.

Este artículo se organiza del siguiente modo: para comenzar se contextualiza la propuesta desde la perspectiva de los modelos teóricos más influyentes. Luego, se analiza la estrategia de desarrollo de la escritura académica desde el punto de vista curricular y pedagógico. Además, se caracterizan los dispositivos pedagógicos (Illera Lobo, 2017; Assis de Figueiredo, 2018; Barceló y Delgado Pugés, 2017; Martínez y Alzate, 2017; Cícero, 2018; Gómez Francisco y Menares Ossandón, 2014; Elgueta Rosas y Palma González, 2014; Quesada, Gómez Ruiz, Gallego Noche y Cubero-Ibáñez, 2019) en implementación en la Escuela de Derecho de la Universidad de Valparaíso. Finalmente, se ofrecen algunas reflexiones finales y se trazan posibles campos de investigación empírica y de innovación académica que pueden ser abordados.

\section{Escribir e interactuar en la Universidad}

En el marco de los estudios de la producción del discurso escrito, el vínculo entre escritura y aprendizaje comienza a ser explorado de manera sistemática en la década de 1970, específicamente a partir de los estudios de Britton (1970) y Emig (1977) en relación con los principios que justifican el carácter único de la escritura como un medio para aprender (Vigotsky, 1934; Klein, Boman y Prince, 2007; Lew y Schmidt, 2011; Navarro, 2018). De esta forma, se inició una serie de reflexiones que enfatizan el potencial epistémico, heurístico y retórico de la composición de textos.

Ahora bien, la relación entre escritura y aprendizaje (López-Gil y Molina Natera, 2018; Álvarez Angulo, Mateo, Serrano y González, 2015; Moyano, 2018; Cabrera Pommiez, 2014; Pan y Litensky, 2018; Castelló, 2015; Fidalgo y García, 2008; Concha, Miño y Vargas, 2017; Lovera y Uribe, 2017) no se limita a actividades de tipo reproductivo que impliquen una mera manipulación del conocimiento ajeno, sino que se trata, tal como lo plantean Miras y Solé (2007), de una tarea de acceso al conocimiento y de elaboración de nuevos conocimientos. Para Emig (1971), por tanto, la escritura se concibe como una particular forma de aprender, ya que posee características que se corresponden con poderosas estrategias de aprendizaje. Esta consideración de la escritura como un modo de aprender ha incentivado el surgimiento de diversas formulaciones teóricas que buscan comprender los procesos cognitivos subyacentes a la producción de discurso escrito (Flower, 1979; Flower y Hayes, 1981; Bereiter y Scardamalia, 2013), así como las características contextuales del escribir como acto social.

Los procesos de lectura y escritura en educación superior en general, y en el ámbito universitario en particular, imponen una serie de exigencias a los sujetos aprendientes (Navarro, 2014). Para dar cumplimiento a estas tareas es necesario sintetizar y organizar información desde múltiples fuentes. Por ejemplo, la escritura de síntesis 
o resúmenes (Parodi, Ibáñez y Venegas, 2014) es un proceso demandante para el estudiante universitario por varias razones. Se trata de una habilidad esencial que requiere un análisis crítico de diferentes fuentes y una elaboración del conocimiento a partir de material textual diverso: artículos, revisiones y libros: «Los estudiantes que tienen que realizar una revisión literaria enfrentan a un cambio importante en perspectiva: de consumidores a productores de conocimiento (Ylijoki, 2001: 21: la traducción es nuestra). Es así que una síntesis de discurso de alta calidad organiza, integra y evalúa los textos fuentes. En contraposición, la investigación en las últimas décadas ha puesto en evidencia cómo los estudiantes repiten secuencialmente el contenido de cada fuente en sus respectivos trabajos sin un análisis ni organización del contenido (Granello, 2001; Segev-Miller, 2007), lo que motiva a la generación de espacios curriculares que posibiliten una iniciación a los procesos de lectura y escritura en el seno de comunidades de práctica específicas.

En este contexto, la comprensión y producción de textos tiene un papel vital en las prácticas de alfabetización avanzada (Sánchez Upegui, 2016; Montijano Cabrera y Barrios, 2016; Carlino, 2017; Aillón Neumann, Figueroa y Neira, 2015; Piacente, 2012; Wadsari, Kristiawan y Arafat, 2019; Davari Torshizi y Bahraman, 2019: Guzmán Simón y García Jiménez, 2017; Cameron, Coleman, Hervey, Rahman y Rostant, 2019; Müller González, 2019): «Debido a que la lectura desempeña un papel tan importante en las tareas de escritura académica avanzada, los educadores se han preocupado cada vez más por la forma en que los escritores durante su desarrollo intentan integrar los textos originales en sus escritos» (Keck, 2014: 4: traducción nuestra). Así, surge la necesidad de saber más acerca de cómo los estudiantes resumen, sintetizan, producen y comparten textos diversos en el ámbito del derecho.

\section{Conjugando dos dispositivos pedagógicos: el curso basal y la mentoría}

El Programa de Aprendizaje Colaborativo (PAC) de la Universidad de Valparaíso (UV) nace a partir del fortalecimiento del Plan de Atención Preferencial a los Primeros Años definido en el Proyecto Educativo de la institución ${ }^{1}$ y busca mejorar paulatinamente los indicadores de progresión y logro por medio de acciones que faciliten el avance académico de todos los estudiantes de primer año de la carreras bajo innovación curricular en la universidad, permitiendo así el desarrollo de competencias transversales y genéricas, como las habilidades sociales, el trabajo colaborativo, la participación activa en la clase, la formación de equipos y grupos de estudio, la inserción social, entre otras. Habilidades todas requeridas para desarrollar un tránsito académico exitoso.

1. Proyecto implementado desde el año 2012 en la Universidad de Valparaíso, disponible en https:// divacad.uv.cl/descargas/proyecto_educativo.pdf. 
De esta manera, los estudiantes de primer año que forman parte de una comunidad de aprendizaje liderada por un mentor (estudiante avanzado de la misma carrera). Estas comunidades de aprendizaje son establecidas desde una coordinación institucional y cada una está formada por grupos de cinco a siete estudiantes más un mentor, los que se reúnen semanalmente para desarrollar un trabajo colaborativo de estudio para todos sus cursos de primer año de carrera. Es así que al interior del curso basal se trabaja de forma colaborativa en comunidades de aprendizaje. De esta forma, cada mentor busca responder a las siguientes competencias genéricas, dentro de las cuales se encuentra la competencia de comunicación académica y profesional:

- Autorregulación y búsqueda continua del mejoramiento de sus conocimientos y habilidades.

- Generación de nuevas ideas y gestionar la información para mejorar su aprendizaje.

- Comunicación efectiva.

De igual forma, los estudiantes mentores (pares disciplinares) también tributan a competencias genéricas decretadas en el PEI UV. Estas son:

- Liderazgo y trabajo en equipo.

- Desempeño en escenarios complejos, con equipos multidisciplinarios y con sentido proactivo.

- Conocimiento, habilidades y actitudes para actuar con sentido de ciudadanía, vocación de servicio público y equidad.

El logro de estas competencias requiere la articulación de diferentes estrategias pedagógicas y didácticas integradas en dispositivos que consideren la variación disciplinar y la identidad peculiar de cada comunidad académica de aprendizaje.

\section{Comunidades de aprendizaje}

Desde hace ya varias décadas, un grupo importante de instituciones de educación superior está iniciando procesos de reforma curricular y reestructurando las aulas con el objetivo de involucrar de forma más activa al estudiante en su aprendizaje (Tinto, 2003; Ramos, Torres, León y Yerbabuena, 2019). En esta línea se inscribe el Programa de Aprendizaje Colaborativo (PAC).

El Programa de Aprendizaje Colaborativo asume como modelo articulador de su propuesta el concepto de comunidades de aprendizaje referidas al aula. Como plantea Coll (2001), este tipo de comunidades de aprendizaje subraya la importancia que en ellas tienen la construcción de un conocimiento colectivo como contexto, plataforma y apoyo a los procesos individuales de aprendizaje. 
Dentro de las características más relevantes de las comunidades de aprendizaje referidas al aula, destacan:

- El acuerdo de hacer progresar el conocimiento y las habilidades colectivas.

- El compromiso con el objetivo de construir y compartir conocimientos nuevos.

- El énfasis en el aprendizaje autónomo y autorregulado, en la adquisición de habilidades y estrategias de aprendizaje metacognitivas y en el aprender a aprender.

- La puesta en marcha de estrategias didácticas de aprendizaje colaborativo.

- La utilización sistemática de estrategias y procedimientos diseñados con el fin de que todos los participantes puedan compartir los aprendizajes.

- La corresponsabilidad de profesores y estudiantes en el aprendizaje.

- La existencia de altos niveles de diálogo, de interacción y de comunicación entre los participantes.

En los términos propuestos por Tinto (2003), el concepto de comunidades de aprendizaje responde a la visión «situada/pragmática-sociohistórica» de la cognición y el aprendizaje que releva tres argumentos fundamentales para la construcción de un marco teórico amplio, integrador y articulado de las comunidades de aprendizaje. En primer lugar, la concepción del aprendizaje como un proceso constructivo en el que los estudiantes son a la vez sujetos y protagonistas de su propio aprendizaje. En segundo lugar, el carácter social del aprendizaje que se apoya en las relaciones interpersonales y que siempre tiene lugar en un contexto determinado y, en tercer lugar, la consideración en torno a que los procesos de desarrollo personal y de socialización y enculturación son complementarios e interdependientes.

En síntesis, las comunidades de aprendizaje se definen como un grupo de personas con diferentes niveles de experiencia, conocimiento y pericia, que aprenden mediante su implicación y participación (Wenger, Trayner y de Laat, 2011), gracias a la colaboración que establecen entre sí, a la construcción del conocimiento colectivo que llevan a cabo y a los diversos tipos de ayuda que se prestan mutuamente.

El concepto de comunidades de práctica refleja también de uno u otro modo las ideas apuntadas. Las comunidades de práctica (Lave y Wenger, 1991 y 2001; Aguilera, Mendoza, Racionero y Soler, 2010) refieren a aquellas relaciones de aprendizaje que se establecen no solo entre un aprendiz y su maestro, sino que también involucran a otros - por ejemplo, a otros aprendices más avanzados-; la actividad de aprender se concibe, entonces, como un esfuerzo comunitario para la resolución de problemas o para la consecución de un fin práctico que considera la interacción grupal.

Las comunidades de práctica (Lave y Wenger, 1991 y 2001) se caracterizan por lo siguiente: 
- Por la existencia de un compromiso mutuo entre subgrupos, que se extienden eventualmente a la totalidad de los integrantes.

- Por compartir una empresa común, cuyas bases son negociables.

- Por tener un repertorio compartido de recursos.

De lo anterior podemos desprender que estas comunidades se crean al interior de las instituciones educativas y se diferencian de la comunidad discursiva (Swales, 2004) básicamente por ser grupos inicialmente abiertos: «sin que necesariamente haya algún tipo de iniciación o incluso conciencia de pertenencia a una comunidad de este tipo» (Harvey y Muñoz, 2006: 98). Ambas nociones son especialmente útiles para comprender cómo al interior del entramado universitario coexisten, convergen y divergen diferentes culturas disciplinares que atraviesan la actividad discursiva de sus integrantes.

Por lo tanto, al interior del curso basal se propician los dos tipos de comunidades: por un lado, las comunidades de aprendizaje ya establecidas por la institución para abordar el primer año de carrera y que poseen una identidad individual y grupal determinada y formas de interacción propias; $y$, por otro, la comunidad de práctica donde el docente del curso forma parte, realizando la tarea de facilitar el aprendizaje no solo a los estudiantes como individuos sino como parte de su propia comunidad de aprendizaje.

\section{Aprendizaje colaborativo}

En esta línea, Elboj y Oliver (2003) definen comunidad de aprendizaje como un proyecto basado en un conjunto de actuaciones dirigidas a la transformación social y educativa de los individuos que forman parte de ella. Es decir, se entiende por comunidad de aprendizaje a un grupo de personas con diferentes niveles de experiencia, conocimiento y pericia, que aprenden entre sí mediante su participación y colaboración en la construcción del conocimiento colectivo y a los diversos tipos de ayuda que se prestan mutuamente. En síntesis, estas comunidades que se crean al interior de las instituciones educativas posibilitan desarrollar las siguientes competencias:

- Aprendizaje colaborativo.

- Debate constructivo y reflexivo.

- Desarrollo de relaciones interpersonales.

- Aprendizaje significativo.

De igual forma, Visser (200o) sostiene que una comunidad de aprendizaje posee una visión y una disposición de aprender. Está dispuesta a interactuar constructivamente con su ambiente, en consecuencia, es una comunidad abierta. Por consi- 
guiente, una comunidad de aprendizaje es el resultado de la transformación social y cultural de un centro educativo, en este caso la universidad y la comunidad académica de derecho; y de su entorno mediante una educación integrada, participativa y permanente, basada en el aprendizaje dialógico (Elboj, Valls y Fort, 200o); objetivo que persigue esta propuesta.

En este sentido, el aprendizaje colaborativo es la construcción del conocimiento a través de la interpretación personal y la validación que el individuo hace del mismo en discusión, argumentación y práctica con sus pares (Cross, 2003). A su vez, Gardner (1985) sugiere que el aprendizaje surge de la participación en actividades compartidas, puesto que el aprendizaje no es estático, pero aparece como consecuencia de la transformación cognitiva resultante de la interacción con otros. En este proceso, el individuo combina las actividades mentales con las prácticas sociales dentro de su comunidad, enriqueciendo de esta manera su proceso de aprendizaje. El aprendizaje colaborativo se valida en el trabajo entre pares o grupal (Nakata, 2006), es decir, los estudiantes practican las técnicas de aprendizaje colaborativo y cooperativo desarrollando trabajos grupales, interactuando con sus pares y participando en actividades de la comunidad de aprendizaje.

\section{El curso basal de competencias comunicativas: ¿Estrategias generales de lectura, escritura y oralidad académicas?}

El curso basal (Ávila Reyes, González-Álvarez y Peñaloza Castillo, 2013) constituye una propuesta mixta que postula que la lectura, la escritura y la oralidad son competencias enseñables en el ámbito universitario y que se pueden desarrollar en los estudiantes «a medida que se los apoya en su ingreso a la comunidad disciplinar, al conocer los géneros y registros que en ella se utilizan» (González-Álvarez, 2016: 140). Este dispositivo se apoya en cuatro ejes: i) metodología basada en proyectos (PBL, por sus siglas en inglés); ii) el estudio de géneros del ámbito especializado jurídico (Coloma y Agüero, 2012); iii) el uso de modelos de procesos para la producción de textos; y iv) el apoyo de pares disciplinares. Estos ejes son contextualizados y adaptados a las necesidades de la disciplina del derecho; el eje de mentorías (apoyo en pares disciplinares) es de gran relevancia en esta propuesta dada su focalización en estudiantes de primer año.

Asimismo, la asignatura apunta a la competencia genérica de egreso, en donde el estudiante actúa como profesional autónomo y responsable en los distintos ámbitos de desempeño, asumiendo como ciudadano sensible y comprometido con su entorno. Dicha competencia genérica comienza a desarrollarse en un primer nivel de logro de acuerdo con el mapa de progreso institucional (desarrollo inicial de la competencia Nivel 1).

El curso de carácter práctico tipo taller contribuye al desarrollo de la primera parte 
de la competencia genérica del perfil UV: «Capacidad para comunicarse en forma oral y escrita en la propia lengua». Desarrolla además distintas competencias que han sido consideradas en el perfil académico profesional, mediante el fortalecimiento de estrategias de producción de textos escritos y orales: ello en el contexto de lo que es constitutivo del fenómeno jurídico, trabajo en equipo y liderazgo en proyectos, dominio de tecnologías de la información, particularmente ofimática y motores de búsqueda, en calidad de usuario de nivel medio, la elaboración de enunciados y cuerpos normativos, propios del derecho, siguiendo las reglas de la técnica de redacción de normas.

Finalmente, de manera transversal al curso, la competencia de autorregulación es intencionada con el fin de aportar a la mejora en el proceso de inducción y adaptación a la vida universitaria. En la tabla 1 se pueden apreciar las competencias específicas y genéricas a las que el curso contribuye en un primer nivel de dominio de la competencia (N1).

Como puede verse en la tabla 1, este curso enfatiza el aprendizaje de la lectura de textos académicos orales, escritos y multimodales, propios del área del conocimiento del derecho. Pretende llamar la atención de los estudiantes sobre la importancia de los procesos de alfabetización académica para el aprendizaje disciplinar y para la adquisición de herramientas para la búsqueda y selección de información, así como el desarrollo de habilidades investigativas que les permitan seguir aprendiendo las prácticas letradas a lo largo de su carrera y sus especialidades.

La base teórica del curso se fundamenta en los modelos interactivos y socioculturales de comprensión de lectura (Solé y Mateos, 2007; Cassany, 2006; Parodi, 2014), en los principios de escritura epistémica y en el modelo de procesos para la producción de textos (Flower y Hayes, 1996), tanto orales como escritos y se adhiere a los principios básicos de los movimientos Escribir en las disciplinas (WID, por su sigla en inglés) y Escribir a través del currículum (WAC, por su sigla en inglés) cuyos desarrollos, de gran éxito en el ámbito universitario, se prolongan por más de cinco décadas en ámbitos académicos de países fundamentalmente anglófonos y francófonos (Bazerman, 2012). A continuación, se presentan los modelos teóricos y didácticos que sustentan la propuesta.

\section{Hacia una plataforma teórica, metodológica y didáctica}

El desarrollo de la alfabetización académica y profesional implica captar la complejidad disciplinar (Aguilar Peña, 2017; Calderon Arévalo y Tapia Ladino, 2016; López, Hernández, Gutiérrez y Zafra, 2015; Pezzeta, 2016; Taranilla y Yúfera, 2012) y desarrollar un trabajo colaborativo con directivos, profesores, asesores, ayudantes y estudiantes. En ese sentido, la propuesta integra elementos de diferentes corrientes, movimientos y modelos teóricos que permitan sustentar una propuesta didáctica vinculada al discurso académico en el área del derecho. A saber: 
Tabla 1. Competencias del currículum de Derecho al que tributan ambos dispositivos pedagógicos.

Competencia(s) a la(s) que colabora
Autorregulación: Manejo de procesos
internos (tanto emocionales, cognitivos
y metacognitivos) y situacionales (acti-
tudinales), que posibiliten al estudiante
guiar, controlar y observar de manera
crítica y activa su propia actuación,
dirigiéndola de manera sistemática hacia
la consecución de metas u objetivos
previamente formulados.

C5. Elaboración de enunciados y cuerpos normativos, propios del quehacer del Derecho, siguiendo las reglas de la técnica de redacción de normas.

\section{Situaciones profesionales Indicadores}

Aplican variedad de estrate- - Reconoce y utiliza las distintas herramientas adquiridas gias para resolver problemas para el logro de una adecuada planificación académica. y siguen plan de acción. - Reconoce y utiliza las distintas estrategias (métodos de estudios) que le permitan afrontar con éxito las tareas académicas.

- Genera planes de acción utilizando diversas estrategias adaptándose a los cambios en el contexto académico.

- Ejecuta correctamente las tareas encomendadas.

- Logra relacionarse con los otros de manera coherente y asertiva.

- Se comunica eficazmente utilizando las herramientas de comunicación eficaz.

Redactar enunciados nor-

C5. N1

mativos simples. Redactar bases para proyectos de ley.

a. Conoce la estructura lógica estándar de los enunciados normativos.

b. Conoce los problemas lógicos y semánticos que pueden afectar a normas o sistemas jurídicos.

c. Reconoce las distintas fuentes del derecho y sus condiciones de validez.

C9. N1

Trabaja en equipo en instituciones públicas o privadas. Lidera en proyectos colaborativos del ámbito jurídico.
Colabora en actividades grupales y en equipos de trabajo organizados por afinidad asumiendo responsablemente el desempeño de las tareas encomendadas por el profesor, según el plan de trabajo que le ha sido entregado.
C10. Dominio de tecnologías de la información, particularmente ofimática y motores de búsqueda, en calidad de usuario de nivel medio 0 avanzado.
Utiliza tecnologías de la información para tareas de representación, asesoría jurídica o investigación en el ámbito jurídico.
C10;.N1

a. Selecciona información científica de calidad en Internet para producir los requerimientos básicos de tareas, trabajos y exposiciones sencillas en procesador de textos, como ejemplo, Microsoft Office.

- Aprender a escribir (Bereiter y Scardamalia, 2013; Flower, 1979): enfoque procesual para el aprendizaje de la escritura y la lectura. Representación de la tarea y aplicación de estrategias cognitivas y metacognitivas para acercarse a los procesos de comunicación académica.

- Escribir para aprender (Carlino, 2005; Castelló, 2007; Klein y Boscolo, 2016): valor epistémico de la escritura. Lectura y escritura de géneros que se leen y escriben en la comunidad disciplinar, en el área y en el curso en específico. Desarrollo de temáticas abordadas en los cursos. Trabajo con múltiples fuentes y construcción de una voz académica y disciplinar. 
- Escribir en las disciplinas (Bazerman, Little, Bethel, Chavkin, Fouquette y Garufis, 2005 y 2016): concepción de la lectura y la escritura como prácticas sociales situadas que exhiben convenciones retóricas propias de cada comunidad. En cada disciplina circulan diferentes géneros discursivos que se utilizan para la co-construcción del conocimiento disciplinar y para transmitir y difundir el conocimiento, para argumentar y convencer (logrando efectos retóricos en su audiencia); en definitiva, para participar en las comunidades académicas, científicas y profesionales.

- Pedagogía basada en género (Swales, 2004; Rose y Martin, 2012; Gardner y Nesi, 2012; Venegas, Núñez, Zamora y Santana, 2015): optimización del grado de inserción en la comunidad de práctica disciplinar y del grado de apropiación de las formas genéricas del ámbito académico, profesional y científico. Considera una reflexión sobre los géneros que se escriben que no se centra exclusivamente en lo cognitivo en un extremo o en los aspectos formales en el otro extremo, sino que considera la complejidad de los discursos científicos, académicos y profesionales.

La figura 1 ilustra de manera sintética los diferentes aportes teóricos y metodológicos que permiten recoger la complejidad de toda propuesta de alfabetización académica que busque potenciar el aprendizaje de los estudiantes y facilitar su incorporación a comunidades de práctica particulares, las que requieren el desarrollo de competencias de lectura y escritura académica que considere la diversidad disciplinar y de géneros discursivos específicos que los estudiantes de derecho deben leer y escribir.

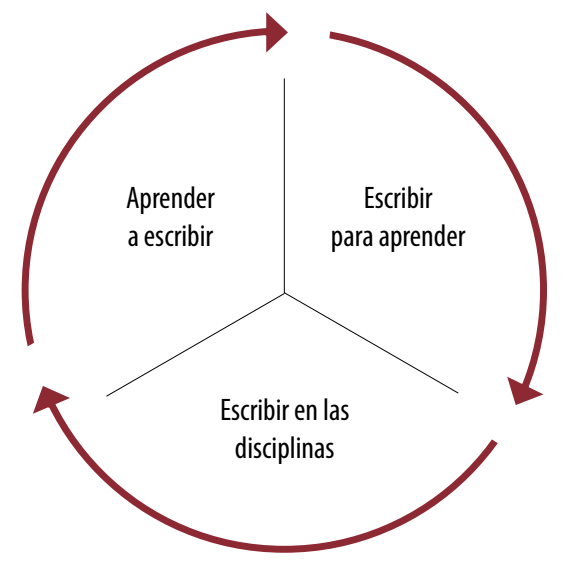

Figura 1. Integración de modelos y corrientes para un programa de lectura y escritura académica y profesional. 
La orientación metodológica se sustenta en el desarrollo de la lectura, de la escritura y la oralidad en prácticas andamiadas en un modelo de transferencia progresiva de la responsabilidad: lectura, escritura, comunicación oral y evaluación de sus prácticas comunicativas de manera conjunta, en pequeños grupos e individualmente (Moyano, 2009). Desde una mirada general, el curso es eminentemente práctico y se estructura didácticamente desde un enfoque contextualista y situado, de modo que las competencias comunicativas se incentivan desde metodologías que acerquen a los estudiantes a las necesidades comunicativas reales del ámbito del derecho y las ciencias jurídicas y sociales. Para ello, se emplean el aprendizaje basado en problemas (ABP) y proyectos, la resolución de problemas (casos) y la formulación de proyectos de investigación disciplinar, escritura y difusión oral del conocimiento disciplinar. Desde una mirada más específica, la comprensión y la producción se trabajan de modo integrado, bajo la premisa teórica de que la primera se evidencia a través de la segunda (Parodi, 2014).

Las tres grandes unidades del curso son las siguientes y siguen el ciclo investigativo de manera de familiarizar a los estudiantes con el desarrollo de un proyecto de investigación/aplicación:

1. Fundamentación y antecedentes para desarrollar una iniciativa de ley (lectura de textos jurídicos y no jurídicos). Etapa de investigación y documentación: se desarrollan estrategias para la comprensión de textos y para la organización de la información. Se usa una secuencia basada en habilidades cognitivas: reconocer información, analizar y evaluar la información verbal con miras a aprender de los textos. Se enseñan explícitamente operaciones resuntivas, fichaje de textos, reconocimiento de múltiples fuentes en un texto académico, protocolos de citado y organizadores de la información.

2. Iniciativa de ley. Etapa de elaboración: esta unidad potencia la escritura de textos como una instancia de aprendizaje e involucramiento con las disciplinas. Se escriben informes bibliográficos, síntesis expositiva, informes de avance y propuesta final (iniciativa de ley, proyecto de ley). En esta etapa toman conciencia del proceso de generación del conocimiento científico, así como del proceso de elaboración de cuerpos normativos.

3. Debate parlamentario. Etapa de difusión: en esta unidad se trabaja en torno a la producción del discurso oral formal y la comunicación oral efectiva. Se prepara un debate parlamentario con base en los resultados de las etapas previas de investigación y elaboración de la iniciativa de ley.

Si bien, cada unidad fortalece una habilidad comunicativa específica, en todas las unidades se desarrolla tanto la comprensión y producción de textos escritos y orales, bajo la premisa de que cada una de las habilidades (leer, escribir, escuchar y hablar) se refuerzan mutuamente; y se utilizan de manera integrada en la comunicación aca- 


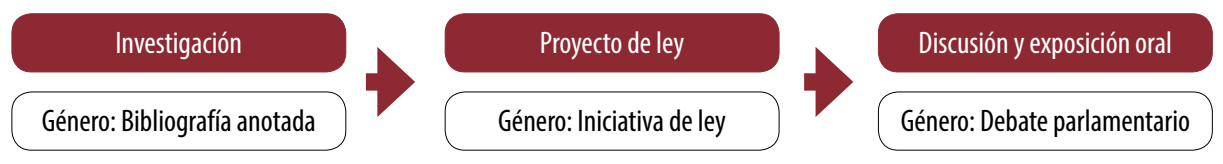

Figura 2. Ciclo de desarrollo del proyecto en el curso basal.

démica y profesional. En la figura 2 es posible apreciar el ciclo a través del cual se organiza el proyecto de la carrera que los estudiantes desarrollan a través del curso.

Este ciclo permite además el andamiaje de las habilidades comunicativas desde géneros discursivos menos complejos y que son parte de géneros más complejos, como la revisión de bibliografía (síntesis discursiva), hacia los textos que requieren la formulación de proyectos (propuesta) y la discusión oral y confrontación de ideas como parte de la deliberación democrática (interacción oral). Los textos de trabajo son seleccionados en conjunto con los profesores del curso Introducción al derecho: órdenes normativos y fuentes del derecho y los estudiantes, a partir de un batería de posibles temáticas configuran su tema en específico, inician búsqueda bibliográfica, luego proceden con la redacción de una bibliografía anotada como género académico de iniciación para posteriormente articularla con la propuesta de ley y revisión de la estructura textual con miras al cumplimiento de los propósitos comunicativos del discurso disciplinar en el ámbito jurídico.

La realización y transformación del aula en una serie de equipos o bancadas que elaboran una iniciativa de ley, permite que los estudiantes desarrollen su conocimiento de los géneros y del lenguaje académico y profesional a partir de las exigencias de la tarea encomendada, lo que permite que se vinculen de forma progresiva con su campo disciplinar y mejoren su motivación y disposición en la utilización de la escritura en todo su potencial epistémico, heurístico, retórico y axiológico. Así pues, con estas competencias comunicativas basales se proyecta una profundización hacia los siguientes ciclos formativos de la carrera de Derecho por medio de la inclusión de espacios curriculares clave que permitan potenciar y evaluar las habilidades de comunicación avanzada. Estos espacios son: Lectura crítica (semestre 2), Taller de integración (semestre 2, 4, 6 y 8), Taller de técnicas de elaboración de normas (semestre 5) y Taller Sello UV (semestre 8, 9 y 10), y permiten dotar de contexto el desarrollo de las competencias de escritura académica y profesional y generar asimismo un programa articulado a través del currículum que evita el aislamiento y la descontextualización en la enseñanza-aprendizaje de competencias fundamentales para el aprendizaje a lo largo de toda la vida. 


\section{Reflexiones finales}

En este trabajo nos propusimos dar cuenta del diseño y la fundamentación del dispositivo pedagógico curso basal de comunicación académica en la Escuela de Derecho de la Universidad de Valparaíso en el marco de la renovación curricular del pregrado y la instalación del nuevo proyecto educativo institucional. En este contexto, el curso basal de la carrera de Derecho se convirtió en un espacio curricular estratégico que logra sintetizar los aspectos clave para la adaptación a la vida universitaria. El desarrollo de la escritura disciplinar, colaborativa y contextualizada permite sentar las bases de lo que será el proceso de aprendizaje indispensable para enfrentar el trabajo académico y profesional.

El diseño del curso, fundamentado en la escritura como herramienta epistémica y en la integración de herramientas que facilitan el dominio de competencias comunicativas en todo el ciclo: lectura, escritura y oralidad, junto al trabajo colaborativo, obtienen adhesión y compromiso con la alfabetización universitaria, lo cual impacta en los aprendizajes disciplinares más complejos a los que se ven expuestos los estudiantes. El aporte de la pedagogía basada en géneros textuales se evidencia en la lectura de textos con los que se acercan a la investigación bibliográfica, comentada y discutida por los equipos de trabajo, con resultados de aprendizaje inspirados en las competencias del nuevo proyecto educativo de la carrera y la universidad. Al enfrentar el género académico son obligados a desplegar una variedad de estrategias de análisis, síntesis y comprensión.

A su vez, el trabajo colaborativo entre profesores del área de derecho (abogados) y profesores especializados en lingüística aplicada (ciencias del lenguaje) permitió una secuencia articulada en torno a una propuesta de «iniciativa de ley», género clasificado dentro del discurso jurídico, lo que acercó a los estudiantes al quehacer de su disciplina, a la discusión de un problema y su respectiva solución. En esta etapa producen sus primeros textos vinculados a su quehacer profesional, pese a que a en este nivel no se les exige dominio de técnica legislativa, pero sigue siendo para estudiantes de primer ciclo un desafío atractivo elaborar una argumentación coherente y con un buen manejo de fuentes.

Es así que todo lo anterior se consolida en el debate parlamentario, donde el ejercicio democrático y deliberativo incentivan una valoración por el trabajo de sus pares y el examen crítico que esto implica. En la constante revisión de sus proyectos descubren que la revisión y edición de sus textos y posterior mejora de ellos es parte del avance en el logro de sus resultados de aprendizaje.

Es interesante destacar que la concepción de enseñanza-aprendizaje de la escritura que promueve el curso basal se centra en una dinámica de interacción entre la comunidad de práctica y las comunidades de aprendizaje, donde el profesor si bien es un experto en su área del conocimiento, también se nutre de la figura del mentor 
como estudiante de curso superior y que se ha apropiado de las prácticas discursivas de su comunidad científica; propuesta de interacción que viene declarada como una política institucional para promover el aprendizaje en colaboración. El mentor también es clave como figura para el estudiante de primer año, pues ratifica la importancia que posee el aprehender las formas discursivas propias de este ámbito del conocimiento. Lo anterior demuestra que las problemáticas y soluciones con respecto a la escritura académica pueden ser abordadas en la universidad no solo por los docentes, sino que también por profesores, profesionales de escritura, estudiantes y la propia universidad (Hermosillo y Verdín, 2019).

La implementación del curso basal en la carrera de Derecho posibilitará al equipo docente del curso indagar sobre cómo los estudiantes de primer año resumen, sintetizan, producen y comparten textos diversos en el ámbito del derecho. Esto se traduce en una propuesta futura de investigación acción en torno a las siguientes preguntas: ¿qué mapas y qué rutas de lectura y escritura activan y cómo este proceso de síntesis se asocia a la comprensión y a la producción, y qué habilidades requieren en contextos de progresiva y creciente especialización, como en el discurso académico y profesional de la comunidad jurídica?

En el diseño y pilotaje de dicho trayecto curricular se han ido incorporando nuevos dispositivos que ayudan a monitorear el trabajo en equipo y los procesos de comprensión y producción. Algunos de ellos son el uso de rúbricas e instrumentos de auto y coevaluación, la elaboración de bitácoras de trabajo en equipo, en el que es posible observar las dinámicas a las que se ven enfrentados. Sin embargo, queda pendiente incorporar algunos apoyos del ámbito de las tecnologías de la información que, pese a que existe una instancia de acercamiento a los distintos motores de búsquedas informáticas, parece no ser suficiente. Resultaría interesante desafiarlos en el trabajo sistemático que se logra en la interacción con aulas virtuales y/u otro tipo de espacios digitales.

Por último, a modo de reflexión, la relevancia de cursos basales como el presentado en este artículo, configurados con dispositivos teóricos que los validan, dan cuenta de esfuerzos que se traducen en experiencias exitosas. Por el contrario, cursos improvisados, sin conocimiento e integración disciplinar, son iniciativas frustrantes y de bajo impacto en la enseñanza superior. La implementación de estos espacios curriculares que actualizan metodologías activas de enseñanza y aprendizaje en educación superior requieren no solo mayor investigación y preparación de los cuerpos docentes, sino que también implican pensar el macrocurrículum (los programas de formación de pregrado) y el microcurrículum (el aula universitaria) de una manera completamente distinta, lo que nos otorga una oportunidad única para desarrollar procesos de investigación-acción y de desarrollo de nuevos recursos didácticos adaptados a la necesidades locales y a la identidad de disciplinas y carreras con una alta heterogeneidad cultural. 
En el contexto de la educación superior chilena, el curso basal se erige como una alternativa viable que no solo concilia formación general y orientación disciplinar, sino que promueve un cambio cultural al involucrar en su quehacer pedagógicos a académicos de la disciplina y a estudiantes avanzados de las mismas comunidades. En efecto, sin su concurso, ambos dispositivos presentados perderían pertinencia. Asimismo, esta propuesta nos acerca hacia lo que efectivamente leen y escriben los estudiantes de la carrera de Derecho, lo que nos permite capturar con un mayor grado de fineza los tipos de interacciones y los desafíos del discurso académico en la academia.

Finalmente, junto con promover nuevos caminos de investigación a lo largo del trayecto formativo, la aplicación de esta metodología nos desafía a implementar y probar nuevos proyectos interdisciplinarios y multidisciplinarios que comiencen en un curso dentro de un semestre y terminen en otro, promoviendo una integración curricular compleja, por ejemplo, a través de propuestas curriculares que posibiliten el diálogo entre carreras de la facultad, entre departamentos, entre estudiantes de diferentes años y experiencias. De esta manera, los abogados en formación pueden movilizar sus competencias de escritura académica en diferentes contextos significativos y relevantes para su práctica profesional.

\section{Referencias}

Aguilar PeÑa, Paula (2017). «Una propuesta de géneros discursivos escritos del ámbito universitario, jurídico y chileno, orientada a la alfabetización académica de estudiantes de derecho». Perfiles Educativos, 39 (155): 179-192.

Aguilera, Antonio, Marlen Mendoza, Sandra Racionero y Marta Soler (2010). «El papel de la universidad en Comunidades de Aprendizaje». Revista Interuniversitaria de Formación del profesorado, 67: 45-56.

Aillón Neumann, Mariana, Beatriz Figueroa y Angie Neira (2015). «Lectura hipertextual en un contexto de alfabetización académica: un estudio de caso». Revista Complutense de Educación, 26: 77-96.

Álvarez Angulo, Teodoro, Teresa Mateo, María del Pilar Serrano y Miguel Ángel González (2015). «Diseño de la plataforma RedacText 2.0 para ayudar a escribir textos académicos e investigar sobre enseñanza y aprendizaje de la escritura». Revista Complutense de Educación, 26 (2): 425-445.

Assis DE FigueIredo, Guilherme (2018) «A aplicação da didática e dos saberes pedagógicos ao ensino superior». Revista de Pesquisa e Educação Jurídica, 4 (2): 18-34. Ávila Reyes, Natalia, Paula González-Álvarez y Christian Peñaloza Castillo (2013). "Creación de un programa de escritura en una universidad chilena: estrategias para promover un cambio institucional». Revista Mexicana de Investigación Educativa, 18 (57): 537-560. 
BArceló, María Tanagua e Iván Delgado Pugés (2017). «Análisis de las subcompetencias lingüística, extralingüística e instrumental en el proceso de enseñanzaaprendizaje de la traducción jurídica y económica (francés-español) a partir de una experiencia docente». Sendebar, 28: 31-51.

Bazerman, Charles, Joseph Little, Lisa Bethel, Teri Chavkin, Danielle Fouquette y Janet Garufis (2005). Writing across the Curriculum. Reference guides to rhetoric and composition. West Lafayette, EEUY: Parlor Press/WAC Clearinghouse.

-. (2016). Escribir a través del currículum. Una guía de referencia. Córdoba: Universidad Nacional de Córdoba.

BAZERMAN, Charles (2012). "Escritura con conceptos: comunales, internalizados y externalizados». Mente, Cultura y Actividad, 19 (3): 259-272.

BEREITER, Carl y Marlene Scardamalia (2013). The psychology of written composition. Hillsdale, NJ: LEA.

Britton, James (1970). Language and Learning. Nueva York: Penguin Books.

Cabrera Pommiez, Marcela (2014). «El instrumento CODICE-Derecho y su aplicación en la Facultad de Derecho de la Universidad de Chile». Revista Pedagogía Universitaria y Didáctica del Derecho, 1 (1): 2-19.

Calderón Arévalo, Edith y Mónica Tapia Ladino (2016). «Sistemas para el apoyo, seguimiento y evaluación de las competencias comunicativas». Revista Mexicana de Investigación Educativa, 21 (69): 411-435.

Cameron, Harriet, Bryan Coleman, Tamara Hervey, Sabrina Rahman y Philip Rostant (2019). «Equality Law Obligations in Higher Education: reasonable adjustments under the Equality Act 2010 in assessment of students with unseen disabilities». Legal Studies, 39 (2): 204-229.

CARLINo, Paula (2005). Escribir, leer, y aprender en la universidad. Una introducción a la alfabetización académica. México: Fondo de Cultura Económica.

Carlino, Paula (2017). «Dos variantes de la alfabetización académica cuando se entrelazan la lectura y la escritura en las materias». Signo y Pensamiento, 36 (71): 18-34.

Cassany, Daniel (2006). Tras las líneas: sobre la lectura contemporánea. Barcelona: Anagrama.

Castelló, Manuel (2007). «El proceso de composición de textos académicos». Castelló, Monserrat (coord.) Escribir y comunicarse en contextos científicos y académicos: conocimientos y estrategias (pp. 47-82). Barcelona: Graó.

Castelló, Monserrat (2015). «La investigación sobre escritura académica en los procesos de enseñanza-aprendizaje en la universidad española». Cultura y Educación: Culture and Education, 27 (3): 471-476.

Cícero, Nidia Karina (2018). «El primer día de clases». Revista de Educación y Derecho, 18: 1-13.

Coll, César (2001). «Las comunidades de aprendizaje y el futuro de la educación: 
el punto de vista del fórum universal de las culturas». En Simposio Internacional sobre Comunidades de Aprendizaje (pp. 1-19). Barcelona.

Coloma, Rodrigo y Claudio Agüero (2012). «Los abogados y las palabras: una propuesta para fortalecer competencias iniciales en los estudiantes de derecho». Revista de Derecho (Coquimbo), 19 (1): 39-69. DOI 10.4067/So718-97532012000100003.

Concha, Soledad, Paola Miño y María Paz Vargas (2017). «Representaciones sociales sobre el conocimiento y la escritura en el pregrado en dos comunidades discursivas: implicancias para la enseñanza de la escritura en la educación superior». Lenguas Modernas, 50: 109-130.

Condon, William y Carol Rutz (2013). «A taxonomy of writing across the curriculum programs: Evolving to serve broader agendas». College Composition and Communication, 64 (2): 357-382.

Cross, Anna (2003). Convencer en clase: Argumentación y discurso docente. Barcelona: Ariel Lingüística.

DAVArI Torshizi, Mehdi y Mastafa Bahraman (2019). «I explain, therefore I learn: Improving students' assessment literacy and deep learning by teaching». Studies in Educational Evaluation, 61: 66-73.

ElвоJ, Carmen y Esther Oliver (2003). «Las comunidades de aprendizaje: Un modelo de educación dialógica en la sociedad del conocimiento». Revista Interuniversitaria de Formación del Profesorado, 17 (3): 91-103.

ElвоJ, Carmen, Rosa Valls y Miguel Fort (2000). «Comunidades de aprendizaje. Una práctica educativa para la sociedad de la información». Cultura y Educación, 12 (1-2): 129-141.

Elgueta Rosas, María Francisca y Eric Eduardo Palma González (2014). «Una propuesta de clasificación de la clase magistral impartida en la Facultad de Derecho». Revista Chilena de Derecho, 41 (3): 907-924.

EMIG, Janet (1971). The composing processes of twelfth graders. Urbana, IL: National Council of Teachers of English.

EMIG, Janet (1977). «Writing as a mode of learning». College Composition and Communication, 28: 122-128.

FidAlgo, Raquel y Jesús García (2008). «El desarrollo de la competencia escrita a través de una enseñanza metacognitiva de la escritura». Cultura y Educación, 20 (3): 325-346.

Flower, Linda (1979). «Writer- Based Prose: A Cognitive Basis for Problems in Writing». College English, 41 (1): 19-37.

FLOwER, Linda y John Hayes (1981). «A cognitive process theory of writing». College Composition and Communication, 32: 365-387.

Flower, Linda y John Hayes (1996). «La teoría de la redacción como proceso cognitivo». En Linda Flower y John Hayes (eds.), Textos en contexto (pp. 3-19). Buenos Aires: Asociación Internacional de Lectura. 
GARDNER, Sheena y Hilary Nesi (2012). «A classification of genre families in university student writing». Applied linguistics, 34 (1): 25-52

Gardner, Robert (1985). Social Psychology and Second Language Learning: The Role of Attitudes and Motivation. Londres: Edward Arnold.

Gómez Francisco, Taeli y Néstor Menares Ossandón (2014).» Los aportes de paradigmas complejos y constructivistas para la enseñanza de la ciencia jurídica». Ius et Praxis, 20 (1): 199-220.

GonZÁlez-Álvarez, Paula (2016). «El curso basal para la enseñanza de la escritura en la universidad». En Cecilia Muse (ed.), Cátedra UNESCO Lectura y Escritura: comunidades, rupturas y reconstrucciones. Vol. 3. Lectura y escritura en el nivel superior (pp. 138-145). Universidad Nacional de Córdoba.

Granello, Darcy Haag (2001). «Promoting cognitive complexity in graduate written work: Using Bloom's taxonomy as a pedagogical tool to improve literature reviews». Counselor Education and Supervision, 40 (4): 292-307.

Guzmán Simón, Fernando y Eduardo García Jiménez (2017). «La alfabetización académica de los futuros maestros. Un estudio comparativo en varias universidades españolas». Revista de Investigación Educativa, 35 (2): 317-335.

Harvey, Ana María (2008). «Acerca de la alfabetización académica y sus manifestaciones discursivas». En Martha Shiro, Haciendo Discurso. Homenaje a Adriana Bolivar (pp. 627-645). Caracas: Universidad Central de Venezuela.

Harvey, Anna María y Daniel Muñoz (2006). «El género informe y sus representaciones en el discurso de los académicos». Estudios Filológicos, 41: 95-114.

Hermosillo, Perla y Paulo Verdín (2019). «Perspectivas de la escritura académica en la universidad: causas, problemáticas y propuestas». Cuaderno de Pedagogía Universitaria, 16(31): 41-57.

ILlERA LoBo, Mercedes (2017). «Relación de la teoría y la práctica en la enseñanza del derecho». Revista Espacios, 38 (45): 1-5.

KeCK, Casey (2014). "Copying, paraphrasing, and academic writing development: A re-examination of $\mathrm{L}_{1}$ and $\mathrm{L} 2$ summarization practices». Journal of Second Language Writing, 25: 4-22.

KLeIn, Perry y Pietro Boscolo (2016). «Trends in research on writing as a learning activity». Journal of Writing Research, 7 (3): 311- 350.

Klein, Perry, Jennifer Boman y Melanie Prince (2007). «Developmental Trends in a Writing to Learn Task» En Writing and Cognition: Research and Applications. Bingley: Emerald.

LAVE, Jean y Etienne Wenger (1991). Situated Learning: legitimate peripheral participation. Nueva York: Cambridge University Press.

-. (2001). «Legitimate peripheral participation in communities of practice». En Supporting Lifelong Learning: Volume I: Perspectives on Learning, (pp. 111-127). Londres: Routledge. 
LEw, Duan Ning Magdeleine y Henk Schmidt (2011). «Writing to learn: can reflection journals be used to promote self-reflection and learning?» Higher Education Research \& Development, 30 (4): 519-532.

Lima, Fernanda y Lucas Noya (2018). «Sentencia simulada del Tribunal Federal Supremo: Aplicar el aprendizaje basado en equipo y la metodología de aprendizaje basada en proyectos en el curso de Derecho». Revista Pedagogía Universitaria y Didáctica del Derecho, 5 (1): 100-111. DOI: 10.5354/0719-5885.2018.50408.

López, Luz, Carlos Hernández, Azael Gutiérrez y Diana Zafra (2015). Efecto de una intervención educativa sobre las competencias comunicativas (Master s thesis, Universidad del Norte).

López-GiL, Karen y Violeta Molina Natera (2018). «Incidencia del trabajo colaborativo docente en la enseñanza y el aprendizaje de la escritura académica». Revista Electrónica de Investigación Educativa, 20(1): 1-13.

Lovera, Pablo y Fernanda Uribe (2017). «Hacia una didáctica crítico-reflexiva en la enseñanza de la escritura en la educación superior». Lenguas Modernas, 50: 91-108.

Martínez, Odette y Luis Alberto Alzate (2017). «Una experiencia para el desarrollo de actitudes del «trabajo en equipos de abogados» para la litigación oral y escrita a través del aprendizaje cooperativo en la asignatura de Derecho Internacional Privado». Revista Pedagogía Universitaria y Didáctica del Derecho, 4 (2): 114-128.

MARINkovich, Juana, Enrique Sologuren y Maha Shawky (2018). "The process of academic literacy in Civil Engineering Computer Science. An approach to academic writing and its genres in a learning community». Círculo de Lingüística Aplicada a la Comunicación, 74: 195-220. http://webs.ucm.es/info/circulo/no74/ marinkovich.pdf. https://dx.doi.org/10.5209/CLAC.60520

Miras, Mariana e Isabel Solé (2007). «La elaboración del conocimiento científico y académico». En Escribir y comunicarse en contextos científicos y académicos (pp. 83-112). Barcelona: Graó.

Montijano Cabrera, María del Pilar y María Elvira Barrios (2016). «Alfabetización académica en la universidad: percepciones de estudiantes sobre una tarea escrita». Opción. Revista de Ciencias Humanas y Sociales, 8: 289-309.

MoyAno, Estela (2009). «Negotiating genre: Lecturer's awareness in genre across the curriculum Project at the university level». En Genre in a changing world (pp. 442264). Colorado/Indiana: Parlor Press \& WAC Clearinghouse.

Moyano, Estela (2018). «La enseñanza de la lectura y la escritura académicas mediante programas a lo largo del curriculum universitario: opción teórica, didáctica y de gestión». Delta. Documentação e Estudos em Linguística Teórica e Aplicada, 34(1): 235-267.

Müller GonzÁlez, Valeska (2019). «Metáfora gramatical ideacional: Un recurso clave para la alfabetización avanzada y académica». Revista Signos, 52 (99): 109-133. 
NaKATA, Yoshiyuki (2006). Motivation and experience in foreign language learning. Bern: Peter Lang: International academic Publisher.

NAVArro, Federico (2014). Manual de escritura para carreras de humanidades. CABA: Editorial de la Facultad de Filosofía y Letras UBA.

Navarro, Federico (2018). «Más allá de la alfabetización académica: las funciones de la escritura en educación superior». En Formação de professores: ensino, linguagens e tecnologías (pp. 13-47). Porto Alegre: Editora Fi.

PAN, Miriam y Andrieli Litenski (2018). "Letramentos e identidade profissional: reflexões sobre leitura, escrita e subjetividade na universidade». Psicologia Escolar e Educacional, 22 (3): 527-534.

PARodi, Giovanni (2014). Comprensión de textos escritos. La teoría de la comunicabilidad. Buenos Aires: Eudeba.

Parodi, Giovanni, Romualdo Ibáñez y René Venegas (2014). «¿Cómo escribir un buen resumen?». Manual de escritura académica y professional. Vol. 2 (pp. 93-119).

Pezzetta, Silvina (2016). «El lenguaje y sus funciones. Conceptos teóricos y aplicaciones en la investigación sobre la enseñanza del Derecho». Academia. Revista sobre Enseñanza del Derecho de Buenos Aires, 14 (27): 131-162.

Piacente, Telma (2012). «Alfabetización inicial y alfabetización académica. Investigación y evaluación de los procesos implicados en el aprendizaje del lenguaje escrito». Revista Iberoamericana de Diagnóstico y Evaluación-e Avaliação Psicológica, 1 (33): 9-30.

Quesada, Victoria, Miguel Ángel Gómez Ruiz, María Beatriz Gallego Noche y Jaione Cubero-Ibáñez (2019). «Should I use co-assessment in higher education? Pros and cons from teachers and students' perspectives». Assessment and Evaluation in Higher Education, 44 (7): 987-1.002.

Ramos, Rómulo, Jessica Torres, Carmen León y Carlos Yerbabuena (2019). «Implementación de comunidades de práctica para el mejoramiento del proceso de enseñanza-aprendizaje de la Geografía en la carrera de Ciencias Sociales de la Universidad Nacional de Chimborazo, Riobamba, Ecuador». Cuaderno de Pedagogía Universitaria, 16 (31): 31-40. https://doi.org/10.29197/cpu.n31.v16.2019.322

Rose, David y James Martin (2012). Learning to write, reading to learn. Londres: Equinox.

Sánchez Upegui, Alexánder (2016). «Alfabetización académica: leer y escribir desde las disciplinas y la investigación». Revista Lasallista de Investigación, 13 (2): 200-209.

SHwom, Bárbara y Penny Hirsch (1999). «Re-envisioning the writing requirement: An interdisciplinary approach». Business Communication Quarterly, 62(1), 104-107.

Segev-Miller, Rachel (2007). "Cognitive processes in discourse synthesis: the case of intertextual processing strategies». In Writing and cognition (pp. 231-250). Netherlands: Elsevier. 
SoLÉ, Isabel y Mar Mateos (2007). «Llegir, pensar i aprendre». Articles de Didàctica de la Llengua i de la Literatura, 41: 15-27.

Sologuren, Enrique (2015). Oralidad académica y metadiscurso: Estrategias discursivas en español. Frankfurt: Editorial Académica Española.

STOLLER, Fredericka (2002). «Project work: a means to promote language and content». En Methodology in Language Teaching. An anthology of current practice (pp. 107-119). Nueva York: Cambridge University Press.

Swales, John (2004). Research genres. Explorations and applications. Cambridge: University Press.

Taranilla, Raquel e Irene Yúfera (2012). «La tipología textual en la enseñanza de la lengua del derecho: Consideraciones a partir de una experiencia docente». Revista de Llengua i Dret, 58: 35-52.

TejedA, Rafael y Pedro Sánchez (2013). «Los proyectos y su contribución en la formación competente de profesionales universitarios». Pedagogía Universitaria, 14 (1): $36-54$.

Tinto, Vincent (2003). «Taking Retention Seriously: Rethinking the First Year of College». NACADA Journal, 19(2): 5-10.

Venegas, René, María Teresa Núñez, Sofía Zamora y Andrea Santana (2015). Escribir desde la pedagogía del género: Guías para escribir el Trabajo Final de Grado en Licenciatura. Valparaíso: Ediciones Universitarias de Valparaíso.

Wandasari, Yulisa, Muhammad Kristiawan y Yasir Arafat (2019). «Policy Evaluation of School's Literacy Movement on Improving Discipline of State High School Students». International Journal of Scientific and Technology Research, 4: 190-198.

Wenger, Etienne, Beverly Trayner y Maarten de Laat (2011). Promoting and assessing value creation in communities and networks: a conceptual framework. Netherlands: Ruud Moor Centrum.

Vigotsky, Lev (2010). Pensamiento y lenguaje. Barcelona: Paidós.

VISEer, Joost (2000). Comunidades de aprendizaje en red (en la construcción de ambientes de aprendizaje para que sean integrales, completos e incluyentes). Presentación llevada a cabo en el Teleseminario sobre Redes de Aprendizaje. Guadalajara, México.

YLIJOKI, Oili-Helena (2001). «Master's thesis writing from a narrative approach». Studies in Higher Education, 26 (1): 21-34.

\section{Reconocimiento}

Este artículo fue escrito en el contexto el proyecto de investigación en docencia de pregrado: «Desarrollo de competencias de comunicación y autorregulación en estudiantes de Derecho de primer año: implementación y evaluación de dispositivos interdisciplinarios», financiado por la Universidad de Valparaíso y desarrollado entre los años 2015 y 2017. 


\section{Sobre los autores}

Enrique Sologuren Insúa es candidato a Doctor en Lingüística Cátedra Unesco para la Lectura y Escritura por la Pontificia Universidad Católica de Valparaíso, Chile. Magíster en Letras con mención en Lingüística y profesor de Castellano, licenciado en Letras Hispánicas y en Educación por la Pontificia Universidad Católica de Chile. Es académico en el Departamento de Ciencia General del Derecho de la Universidad de Valparaíso y Profesor del Instituto de Literatura de la Universidad de los Andes. Su correo electrónico es esologuren@miuandes.cl. (D) https://orcid. org/0000-0001-7803-1817.

Cristina Bonifaz Reyes es magíster en Comunicación y Periodismo por la Pontificia Universidad Católica de Valparaíso y profesora de Castellano por la Universidad de Santiago de Chile. Es profesora en el Departamento de Ciencia General del Derecho de la Universidad de Valparaíso y secretaria ejecutiva del Comité Curricular de la Escuela de Derecho de la misma universidad. Su correo electrónico es cristina. bonifaz@uv.cl. (D) https//orcid.org/oooo-0002-1553-0261.

Carmen Gloria Núñez Castillo es magíster en Lingüística Aplicada y profesora de Lenguaje y Comunicación por la Pontificia Universidad Católica de Valparaíso, Chile. Es asesora pedagógica del Programa de Alfabetización Académica y Profesional de la Escuela de Ingeniería y Ciencias de la Universidad de Chile y profesora en el Departamento de Lingüística de la Universidad de Playa Ancha, Chile. Su correo electrónico es carmen.nunez@uchile.cl. (D) https//orcid.org/oooo-0001-7534-6952. 
La Revista Pedagogía Universitaria y Didáctica del Derecho (RPUDD) es una publicación científica semestral que contribuye a la reflexión multidisciplinaria sobre pedagogía universitaria y didáctica del derecho, para la formación y consolidación de esta área de investigación; así como a la difusión de prácticas innovadoras en la enseñanza-aprendizaje del derecho considerando el contexto nacional e internacional. Es una publicación electrónica internacional con una codirección entre Brasil y Chile.

\author{
DIRECTORA \\ María Francisca Elgueta Rozas \\ Universidad de Chile \\ DIRECTOR \\ Renato Duro Dias \\ Universidad Federal de Rio Grande, Brasil \\ SITIO WEB \\ pedagogiaderecho.uchile.cl \\ CORREO ELECTRÓNICO \\ rpedagogia@derecho.uchile.cl \\ LICENCIA DE ESTE ARTÍCULO
}

Creative Commons Atribución Compartir Igual 4.o Internacional

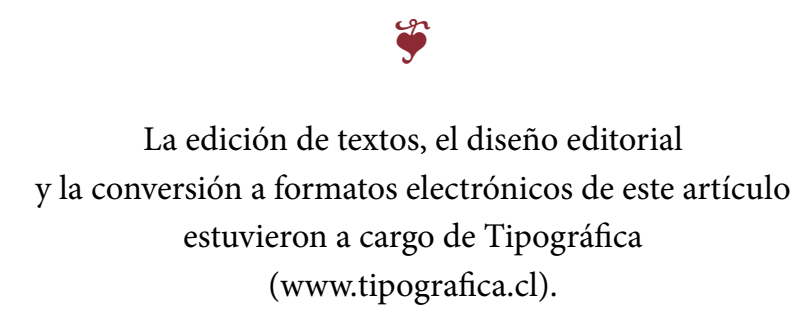

